\title{
Les évolutions récentes du droit public économique sénégalais
}

\author{
Ibrahima Diallo*
}

\section{Résumé}

Cette étude porte sur les évolutions récentes du droit public économique sénégalais. En effet, compte tenu de l'importance des interventions publiques dans l'économie sénégalaise et de la complexité croissante des problèmes juridiques qu'elles posaient, il est estimé nécessaire d'étudier les règles juridiques qui encadrent ces différentes actions qui caractérisent le droit public économique sénégalais c'est à dire l'étude des mécanismes de l'action des pouvoirs publics sur l'économie qui permet de disposer aujourd'hui d'un panorama complet de l'organisation de l'administration économique sénégalaise et des conditions de mise en œuvre d'intervention à caractère économique.

\section{Introduction}

La prégnance de l'action des entreprises ou personnes publiques sur le marché n'a pas été sans susciter et soulever d'importants problèmes en droit public économique sénégalais. En effet, l'action publique sénégalaise en matière économique et toute tentative de normalisation en la matière se heurtent au caractère imprévisible et fluctuant des lois économiques. ${ }^{1}$ Ceci n'empêche pas pour autant l'Etat sénégalais de réglementer certains secteurs ou certaines activités. Il n'en demeure pas moins vrai que cet encadrement normatif, non seulement se caractérise par sa singularité voire par son originalité, mais encore se doit constamment d'être réadapté à la situation économique qui, elle, par définition ne reste figée en aucune façon. Ce trait d'originalité dont il est question caractérise en fait l'ensemble du droit public économique sénégalais. ${ }^{2}$

En droit sénégalais, l'entreprise publique a toujours occupé une place importante dans les mécanismes juridiques mis en place par le législateur pour atteindre les objectifs de développement économique. En effet, comme la plupart des pays en voie de développement, les exigences de la société moderne en matière de service public, de régulation du marché, de promotion d'un environnement plus favorable aux investissements, d'infrastructures si

* Dr. Ibrahima Diallo, Université Gaston Berger, Saint Louis, Sénégal.

1 Bazex, M., Vers de nouveaux modèles normatifs pour le secteur public? AJDA, 1990, 659.

2 Voir Bazex, M., A la recherche de l'autonomie des Entreprises Publiques, Actualités de l'EP, juin 94, 246; voir Bellito, M. Histoire des Entreprises publiques au Sénégal, Harmattan, 2002. Voir Dufau, J., Remarques sur la notion d'entreprise publique, AJDA, 1956, 89. Voir Dufau, J., Les Entreprises publiques, Ed. du Moniteur, 1991. Voir Mescheriakoff, A.-S., L'Autonomie des entreprises publiques, R.D.P, 1990,1575. 
l'on connaît la faiblesse des Etats d'Afrique subsaharien, en l'occurrence le Sénégal, en matière de structures d'équipement collectif, ont caractérisé l'orientation des politiques publiques de ces dix dernières années. ${ }^{3}$

Les questions auxquelles l'Etat devra apporter des réponses concrètes ne se posent plus dans les mêmes termes. ${ }^{4}$ Et de nouveaux problèmes surgissent brutalement et prennent une prégnance qui requiert, au risque de laisser le droit en marge de cette évolution, des adaptations juridiques. ${ }^{5}$ Le secteur économique est le plus touché par cette nouvelle donne. La constitution d'espaces économiques d'intégration telles que l'Union Economique et Monétaire Ouest Africain (UEMOA) exige des Etats africains l'adaptation de leurs législations mais aussi de leur environnement administratif. Ainsi, la permanence des enjeux d'efficacité, de financement, de responsabilisation, et de légitimité conduit à la mise en place de mécanismes d'externalisation des services qui se traduit dans les faits par la création d'entreprises publiques. Il s'agit des réponses élaborées pour répondre à la crise de l'État-providence, plus précisément à la crise du service public dans les Etats modernes. Cette crise correspondrait, contrairement à ce que l'on pourrait croire, moins à un désengagement pur et simple de l'Etat du secteur économique, qu'à un redéploiement, une modification en profondeur de ses modes d'intervention.

La question fondamentale est de retracer les évolutions actuelles de droit public économique sénégalais. L'intérêt de cette question est lié au fait que l'administration sénégalaise, à l'instar de celle de la plupart des pays en voie de développement, se caractérise par des dysfonctionnements chroniques entraînant une inefficacité des interventions publiques. Ces dysfonctionnements sont le produit d'une part, du cadre juridique qui régit la vie quotidienne des administrations et d'autre part, de carences dans la gestion des affaires publiques. Aussi, la réforme du droit des entreprises publiques ne relève pas du hasard. Il s'inscrit dans un contexte qui nécessitait la mise en place d'institutions performantes et aptes à répondre aux défis du développement. Cela suppose la redéfinition des rôles des différentes institutions, leur structuration, ainsi que les voies et moyens permettant de répondre aux attentes de la population en matière de bien-être et de service public. Au Sénégal, puisque les principales interventions publiques sur les activités économiques se présentent généralement sous des formes complexes, il ne semble pas inutile de tenter d'établir

3 Eod. loc.

4 Nzouankeu, J-M., Les contrats in remarques sur les particularités de droit administratifs sénégalais, RIPAS n ${ }^{\circ}$ 9, p. 3. Voir aussi Ngaïde, M., La notion de matière administrative au Sénégal, RASDP n ${ }^{\circ} 5,6,7,8$ de 1998, p. 147.

5 Sy, D., L'évolution du droit administratif sénégalais, Revue EDJA, $\mathrm{n}^{\circ}$ 67, trimestriel (Oct., Nov., Déc. 2005), p. 40; Gaye, Oumar / Mamadou Diouf, Le Conseil d'Etat et la pratique du recours en annulation, édition 2001, Dakar, NIS; LAVROFF, D.G., Le code sénégalais des obligations de l'administration, Recueil PENANT 1966, p. 6 à 4; Diop, Serigne, Quelques remarques sur le code des obligations de l'administration au Sénégal, RIPAS n¹6, janvier-juin 1987, p.100 à 101. 
une présentation synthétique des problèmes juridiques que soulèvent les interventions publiques en matière économique. ${ }^{6}$

Par ailleurs, l'ignorance du passé ne se borne pas à nuire à la connaissance du présent mais elle compromet, dans le présent, l'action même. Il n'est pas possible de comprendre les enjeux actuels du droit public des activités économiques sans en connaître les origines, ni sans étudier les débats théoriques qu'a pu susciter, dans l'histoire du Sénégal, la question des rapports entre l'Etat et le marché. La notion même de droit public des activités économiques apparaît corrélée à celle d'interventionnisme économique. L'importance de ce droit est ainsi fonction de niveau d'implication de l'Etat et des personnes publiques dans l'économie. Or les rapports entre l'Etat et l'économie se sont révélés particulièrement mouvants et évolutifs. Dès 1956 et, plus encore, après l'indépendance de 1960, toute la nouvelle administration sénégalaise n'a d'autres solutions que de se bâtir sur les cendres même de l'administration coloniale française qui l'a précédée. Qu'il s'agisse des structures organiques, des règles de fonctionnement ou du statut des personnels, les emprunts sont très nombreux. Ils sont rendus nécessaires par l'obligation faite à la jeune République du Sénégal d'asseoir rapidement son pouvoir et sa légitimité. ${ }^{7}$

6 Colson, J.-P., Droit public économique, $3^{\mathrm{e}}$ éd., LGDJ; Cliquenois, M., Droit public économique, 2001, Ellipses; Hubrecht, H.G., Droit public économique, 1997, Dalloz; Vlachos, G., Droit public économique français et communautaire, 2000, Dalloz.

7 Le secteur parapublic ne fait pas exception et les premiers établissements sortent du portefeuille détenu par la défunte administration territoriale. Après l'office des H.L.M. qui a succédé à l'O.H.E. (Office des Habitations Economiques), les deux grands services publics que sont les chemins de fer et le Port Autonome, sont les premiers à rejoindre les rangs du tout nouveau secteur parapublic national, dont ils constituent l'ossature. A ces grands services parapublics viennent s'adjoindre des transfuges de l'ancienne administration, qui accèdent enfin à l'autonomie. C'est le cas de l'office des postes et télécommunications et la Régie des transports du Sénégal qui s'occupent d'activités à caractère commercial. Enfin quelques sociétés d'économie mixtes viennent compléter le dispositif d'offres de services publics. La SICAP fait partie de l'héritage colonial. Elle intervient dans le domaine de l'aménagement urbain et de l'habitat en complément l'O.H.L.M. Pour le reste, on assiste à la constitution de nouvelles sociétés qui viennent appuyer l'action du gouvernement dans les domaines de télécommunications et des transports collectifs. Ainsi, Tele Sénégal (Télécommunication du Sénégal) est créée en 1918 pour s'occuper de la gestion des lignes internationales. Elle a été précédée par la COSENA (Compagnie Sénégalaise de la Navigation) développée trois années plus tôt pour prendre en charge la navigation maritime et par la compagnie aérienne transcontinentale Air Afrique dans laquelle le Sénégal a pris une participation minoritaire significative. Si le contrôle de grands services publics, qui sont les garants même de l'unité du pouvoir et de la stabilité économique d'un pays, constitue l'aspect le plus visible de l'affirmation de son pouvoir, l'Etat ne s'arrête pas en si bon chemin et s'assure également une maîtrise réelle de l'information. Le statut d'établissement public à caractère administratif attribué à l'ORTS, en 1966 semble trahir la volonté du pouvoir de ne pas concéder trop d'autonomie à une activité jugée sensible. A la fin des années 60 , la structure du secteur parapublic sénégalais permet de dégager quatre grands rôles d'activité :

un secteur rural (partie Nord-Ouest) dominant constitué autour de l'ensemble OCA (Office de Commercialisation Agricole)-ONCAD (Office National de Coopération et d'Assistance au Développement) (arachide) et quatre sociétés de développement régional : SODENIA (Société de Développement de la région des Niayes du Sénégal), SDRS (Société de Développement Rizicole du Sénégal) SAED et SODAICA (Société de Développement Agricole et Industrielle de la Casamance). Ce 
Le secteur parapublic ne fait pas exception et les premiers établissements sortent du portefeuille détenu par la défunte administration territoriale. ${ }^{8}$

Toutefois, les fondements et les conditions d'existence du secteur public ont, en effet, été bouleversés au cours des quinze dernières années par la conjugaison dynamique des explosions techniques, des libéralisations et de la modification des rapports sociaux. ${ }^{9}$ Ainsi nous avons assisté à une situation pouvant être décrite de déclin et face à cette crise des modalités de réformes ont été mises en place. En résumé, les maux du secteur parapublic au Sénégal, comme du reste partout ailleurs en Afrique, ont été ainsi diagnostiqués en terme de pléthore de personnel et le recours chronique aux subventions de l'Etat pour combler les déficits; cela a été particulièrement le cas des entreprises publiques du monde rural, qui, à elles seules, recueillent 60 pour cent du total des subventions.

Pour le Sénégal, les déficits étaient très importants. La grande sécheresse, le choc pétrolier, les déficits ont posé l'exigence de redéfinition du secteur parapublic. Un plan de sensibilisation avait été proposé et couvrait la période de 1978-1979 et sera suivi par un autre

noyau est essentiellement le fait d'établissement public. Ces secteurs sont renforcés par des SEM comme la SERAS (élevage), SOSA Pêche et COMAPECHE (pêche), SIV (textiles), SEBA (transformation du bois)

un secteur bancaire d'Etat (partie centrale) qui se veut être l'instrument gouvernemental de la politique de développement, grâce notamment à sa politique de financement du monde rural (BNDS) et de mobilisation de l'épargne nationale (USB).

L'urbanisme (partie Est) avec une action que s'appuie exclusivement sur deux structures de nature juridique différentes : OHLM (établissement) et une SEM, la SICAP.

Enfin, de grands services publics interviennent en matière de transports (RCFS, COSENA) et de communications (ORTS, OPT, APS).

Ces établissements publics assurent le lien entre les différentes régions du Sénégal et des garants de l'unité nationale. Quelques établissements publics à caractère administratif et professionnel consacrés à l'action sociale (CSS, ONAC, COND) et à l'animation du monde rural (ITA, SEMA) apportent la touche finale à ce dispositif à la fin des années 1960 .

8 Après l'office des H.L.M. qui a succédé à l'O.H.E. (Office des Habitations Economiques), les deux grands services publics que sont les chemins de fer et le Port Autonome, sont les premiers à rejoindre les rangs du tout nouveau secteur parapublic national, dont ils constituent l'ossature. A ces grands services parapublics viennent s'adjoindre des transfuges de l'ancienne administration, qui accèdent enfin à l'autonomie. C'est le cas de l'office des postes et télécommunications et la Régie des transports du Sénégal qui s'occupent d'activités à caractère commercial. Enfin quelques sociétés d'économie mixtes viennent compléter le dispositif d'offres de services publics. La SICAP fait partie de l'héritage colonial. Elle intervient dans le domaine de l'aménagement urbain et de l'habitat en complément l'O.H.L.M. Pour le reste, on assiste à la constitution de nouvelles sociétés qui viennent appuyer l'action du gouvernement dans les domaines de télécommunications et des transports collectifs. Ainsi, Tele Sénégal (Télécommunication du Sénégal) est créée en 1918 pour s'occuper de la gestion des lignes internationales. Elle a été précédée par la COSENA (Compagnie Sénégalaise de la Navigation) développée trois années plus tôt pour prendre en charge la navigation maritime et par la compagnie aérienne transcontinentale Air Afrique dans laquelle le Sénégal a pris une participation minoritaire significative.

9 Voir notamment Lombard, M., A propos de la concurrence entre opérateurs publics et opérateurs privés, D. 1994, I, p. 163. 
plan: le Plan de Redressement Economique (1979 - 1985). ${ }^{10}$ Depuis 1985, l'Etat sénégalais poursuit des objectifs précis dans une logique de désengagement progressif. Ce désengagement va affecter toute l'activité économique de l'Etat. ${ }^{11}$ Il constitue l'une des axes de la nouvelle politique économique. ${ }^{12}$ Devant cette situation, les pouvoirs publics ont initié des réformes allant dans le sens de la réorganisation du secteur parapublic sénégalais. Aujourd'hui, nous assistons à une refondation du droit public économique sénégalais reposant non seulement l'extension des institutions et champs d'intervention mais aussi par la mise en place une régulation économique rénovée. ${ }^{13}$ En outre, cette régulation économique ${ }^{14}$ est surtout encouragée par le droit communautaire issu de l'UEMOA régit en partie l'espace économique de ses Etats membres notamment celui du Sénégal. De ce fait quand l'Union

10 A partir de 1985, il y a eu une nouvelle mise en œuvre d'un nouveau cadre de relance de l'économie sénégalaise. Les remèdes n'eurent pas les effets escomptés. En 1987, une autre étape sera franchie avec la loi sur la privatisation. Aussi, le service public a fait l'objet d'un harcèlement textuel comme on peut le remarquer en revisitant les remaniements des dispositifs juridiques et institutionnels.

11 Vlachos, G., Droit public économique français et communautaire, A. Colin, 2001, 414 p.

12 Il faut préciser que pour les autres entités appartenant au secteur parapublic, il s'était agi pour l'Etat de se retirer du secteur marchand ou l'initiative privée était jugée plus performante, à réduire le coût du secteur pour les finances publiques. Concernant le secteur parapublic, deux (02) directions ont été prises:

Le désengagement de l'Etat du capital des sociétés ou des entreprises publiques. Les entreprises concernées par cette mesure sont celles qui ont une activité commerciale, celle non viable et vouées à la liquidation. Enfin les entreprises qui ont atteint les objectifs assignés qui leur avaient été fixées initialement et dont le maintien dans le secteur parapublic ne se justifiaient plus.

La réduction des subventions directes et indirectes qui peut-être regardée comme le $2^{\mathrm{e}}$ volet du désengagement. Malgré l'importance des efforts consentis, la charge ou déficit jusqu'en 1991 ne décroît pas et l'explication n'est pas à rechercher dans les défaillances de l'administration réputée peu préparée à la gestion des entreprises publiques. Il faut avec Marc Bellito accepter que la logique expansionniste a toujours prévalu « et que l'action politique a toujours pris le pas sur les exigences d'une dynamique réformatrice ».

13 Avec la mondialisation, on tend de plus en plus vers une économie mondiale. Mais si cette dernière est devenue effective, demeure le fait qu'une gouvernance mondiale, ou à tout le moins quelque chose qui y ressemblerait n'existe pas encore. Si cette interdépendance peut jouer en faveur de certains Etats, il semble que compte de la concurrence très rude auquel les économies se livrent sur le plan mondial, celles des pays en voie de développement comme le Sénégal ne soient pas encore suffisamment outillées pour y faire face. Ainsi se voient justifier la mise en place d'une certaine législation économique communautaire qui n'est pas sans influence sur les politiques d'ouverture au marché qui, s'exerçant toujours à l'échelle des Etats n'en demeure pas moins poreuse aux mutations juridiques sous-régionales voire internationales.

14 Dans un sens assez littéral, le terme de régulation est désormais régulièrement utilisé dans la sphère économique et des politiques publiques pour désigner tout ce qui est fait pour apporter par une action appropriée à la marge, une stabilité satisfaisante à un système économique ou même social. Il s'agit donc de la recherche d'un certain équilibre effectuée à travers la mise en œuvre de divers mécanismes. La récente vogue que connaissent ces institutions surtout dans le cadre sénégalais, témoignent de l'intérêt que suscitent la régulation économique dans nos jeunes Etats. Il semblerait d'ailleurs que régulation et autorités de régulation soient consubstantiellement liées. 
incite les Etats membres à coordonner leurs politiques sectorielles nationales dans certains domaines où existent des obligations de service public notamment ceux des télécommunications, des transports ou de l'énergie, elle vise aussi inéluctablement la matière de la régulation des services en réseaux. ${ }^{15}$ Mais c'est l'Union qui va définir le cadre en édictant des règles communes à l'ensemble des Etats membres. En effet, vu l'explosion du secteur et les nombreuses possibilités de croissance qu'il offre, nos Etats se sont très tôt lancés dans une politique concurrentielle. ${ }^{16}$

Les interventions économiques de l'Etat sénégalais s'inscrivent dans une démarche normative, marquée par l'importance du principe de libre concurrence et par le renoncement aux procédés les plus autoritaires de direction de l'économie. Toutefois, le principe de libre concurrence n'empêche aucunement l'intervention des personnes publiques sur le marché. C'est donc davantage à une mutation qu'à une disparition de l'interventionnisme économique que l'on assiste depuis les années 1980. Cette mutation se manifeste par l'émergence d'un actionnariat public stratégique, par la valorisation de la fonction de régulation de l'économie, par le développement du recours aux partenariats et, enfin, par l'émergence de nouveaux acteurs infra et supra étatiques. Il ne faut pas déduire trop rapidement des nombreuses privatisations d'entreprises intervenues depuis plus d'une vingtaine d'années que l'Etat sénégalais se serait désengagé de l'économie ou qu'il aurait renoncé à mener une politique industrielle dans l'intérêt économique national. Encore présent au capital de nombreuses entreprises, l'Etat dispose parfois d'actions spécifiques qui font de lui un actionnaire privilégié, lui permettant, par exemple, de s'opposer à des modifications de capital ou à la cession par l'entreprise privatisée de certains actifs stratégiques. ${ }^{17}$ Ainsi, l'Etat sénégalais se voit investi dans le domaine économique. Il devient le gardien des grands équilibres économiques (croissance, inflation, chômage, balance des paiements) et le garant de la soli-

15 Orsoni, G., L'administration de l'économie, LGDJ, 1995, 194 p.

16 Directive [01]/2006/CM/UEMOA relative à l'harmonisation des politiques de contrôle et de régulation du secteur des Télécommunications; Directive [02]/2006/CM/UEMOA relative à l'harmonisation du régime applicable aux opérateurs de réseaux et fournisseurs de services; Directive [03]/ 2006/CM/UEMOA relative à l'interconnexion des réseaux et services de Télécommunications; Directive [04]/2006/CM/UEMOA relative au service universel et aux obligations de performances du réseau; Directive [06]/2006/CM/UEMOA organisant le cadre général d'une coopération entre les Autorités Nationales de Régulation (ANR) en matière de Télécommunications. Celles-ci furent adoptées le 23 mars 2006 à Abidjan. Dans la même dynamique, la CEDEAO a pris des actes additionnels en l'occurrence l'Acte Additionnel A/SA 6/01/07 relatif à l'accès universel/service universel. Dans le domaine des marchés publics on peut souligner la Directive $\mathrm{N}^{\circ}$ 05/2005/CM/ UEMOA portant contrôle et régulation des marchés publics et des délégations de service public dans l'Union économique et monétaire ouest africaine.

17 Par ailleurs, l'Etat exerce une influence déterminante, au moment de la privatisation, sur la composition du capital des entreprises et tente de mettre ses " champions nationaux " en situation de mieux affronter la concurrence internationale afin de préserver son influence sur les choix de l'entreprise. Plus ponctuellement, les autorités étatiques font pression sur les entreprises pour infléchir leurs choix, notamment pour les inciter à maintenir leurs unités de production sur le territoire national. 
darité nationale (protection contre les risques sociaux, mise en œuvre de la solidarité nationale). L'interventionnisme économique de l'Etat sénégalais se manifeste, d'abord, par l'extension considérable du secteur public marchand. En effet, le secteur parapublic au Sénégal est régi par la loi 90-07 du 26 juin 1990 mais d'autres textes interviennent pour règlementer certains secteurs de la vie des entreprises publiques. Mais ces textes ne définissent pas l'entreprise publique. Ils font apparaître d'ailleurs un nouveau glissement terminologique comme le secteur parapublic, ce qui pourrait impliquer que le secteur parapublic est plus vaste que les entreprises publiques. La loi de 1990 précitée consacre une notion évolutive du secteur parapublic. II résulte de ces éléments, qu'aujourd'hui, le droit public économique sénégalais peut être appréhendé sous l'angle du double rôle joué par l'Etat en tant que acteur et régulateur. Sous l'angle de l'intervention de l'Etat sénégalais en tant qu'acteur, nous notons un élargissement juridique de son champ d'intervention (I). Sous l'angle de l'intervention de l'Etat sénégalais en tant que régulateur, nous observons une valorisation juridique de la régulation économique (II).

\section{Un champ d'intervention juridiquement élargi}

Aux termes de la loi 1990, le secteur parapublic comprend les établissements publics à caractère industriel et commercial, les sociétés nationales et les sociétés anonymes à participation publique majoritaire. Parallèlement à la loi de 1990 sur le secteur parapublic, le législateur a adopté une loi d'orientation sur les agences. ${ }^{18}$ En effet, la complexité des procédures administratives et l'exigence accrue des usagers du service public en termes de célérité et d'efficacité ont conduit l'Etat sénégalais à créer, dans plusieurs secteurs d'activités (investissement, assainissement, distribution, tourisme, sécurité, technologies de l'information et de la Communication), des organes décentralisés sous des appellations multiples (agences, autorités, office, conseil, etc.). Cette loi vise à harmoniser et à encadrer la pluralité et la diversité des modes de création, d'organisation et de fonctionnement des agences. Il ressort de ces éléments un élargissement juridique du cadre d'intervention de l'Etat dans l'économie sénégalaise. Ainsi, deux acteurs majeurs d'intervention peuvent être retenus dans le domaine économique: Les entreprises du secteur parapublic (A) et les agences d'exécution (B).

\section{A. Les entreprises du secteur parapublic}

La notion d'entreprise publique est très difficile à définir. La loi 90-07 du 26 juin 1990 relative à l'organisation et au contrôle des entreprises du secteur parapublic ne donne pas une définition de l'entreprise du secteur parapublique, il se contente juste de préciser que le secteur parapublic comprend les établissements publics industriels et commerciaux, les sociétés nationales, les sociétés anonymes à participation publique majoritaire. Il est nécessaire

18 Loi d'orientation, n 2009-20 du 4 mai 2009, sur les agences d'exécution. 
de définir les différentes catégories juridiques qualifiées par la loi de $1990^{19}$ comme étant les entreprises du secteur parapublic avant toute appréciation juridique.

Tout d'abord, nous avons les établissements publics industriels et commerciaux qui sont des institutions spécialisées dotées d'un patrimoine propre et de l'autonomie financière et ne bénéficiant d'aucun apport privé à leur fond de dotation. Leur champ d'intervention est varié. Ils peuvent intervenir dans différents domaines scientifique, culturel. ${ }^{20} \mathrm{Ils}$ ont l'autonomie de gestion et s'administre librement sous la responsabilité de leur organe délibérant, sous réserve des contrôles prévus. Les établissements publics industriels et commerciaux différent des établissements publics administratifs et établissements publics professionnels même si la différenciation n'est pas toujours évidente. L'établissement public industriel et commercial constitue le support juridique classique de l'entreprise publique, celui qui connaît aussi un déclin marqué dans la période contemporaine. Né de la conjonction de l'établissement public et du service public industriel et commercial, l'établissement public industriel et commercial appelé EPIC ne s'est pas cantonné dans la gestion des SPIC. Il est apparu pour l'Etat comme un nouveau moyen d'action se démarquant des modes d'intervention exclusifs, direct et indirect que la régie et la concession constituaient respectivement jusqu'alors. Par rapport à la régie, l'EPIC offre en premier lieu l'avantage comme tout établissement public, de posséder une personnalité juridique propre, une autonomie financière et un patrimoine différents de celui de sa collectivité de rattachement. Cependant il demeure une personne morale de droit public, gage de son appartenance au secteur parapublic et le caractère public de son patrimoine lui assure une protection particulière. Enfin son caractère industriel et commercial offre une possibilité d'adaptation de cette forme juridique aux exigences de la commercialité. Le recours à l'EPIC n'est pas exclusif à l'utilisation de la concession pour la gestion d'un service public. Seulement, s'il existe, il en dénature la signification et l'économie traditionnelle en substituant au concessionnaire privé une personne publique. $^{21}$

Ensuite, nous avons les sociétés nationales qui sont prévues à l'article 4 de la loi de 1990 qui les définit comme des sociétés par action de droit privé dont le capital est intégralement souscrit par l'Etat et le cas échéant par d'autres personnes morales de droit public. Dans tous les cas, la participation directe de l'Etat est supérieure à $50 \%$ du capital social. Donc, il s'agit d'une entreprise dont le capital est détenu par l'Etat ou ses démembrements et pour laquelle le statut de droit privé est maintenu. L'objectif recherché par l'Etat est de placer sociétés nationales dans des conditions d'organisation identiques à celles du secteur privé. En définitive, sociétés nationales sont des sociétés anonymes dont l'Etat est l'unique actionnaire. Cette forme a servi de support direct à la création des entreprises publiques issues de la nationalisation d'entreprises dont on souhaitait seulement modifier le régime

19 La loi 90-07 du 26 juin 1990 relative à l'organisation et au contrôle des entreprises du secteur parapublic.

20 Ils sont créés par la loi et leur règle d'organisation et de fonctionnement sont prévus par décret.

$21 D u f a u$, J., Les Entreprises publiques, Ed. du Moniteur, 1991. 
d'appropriation. La forme juridique des sociétés nationalisées offre une souplesse de gestion et permet une meilleure adaptation du secteur public aux exigences croissantes de la concurrence telles qu'elles se manifestent au niveau communautaire.

Enfin, nous avons les sociétés anonymes à participation publique majoritaire dans lesquelles une ou plusieurs personnes publiques possèdent directement ou indirectement au moins $50 \%$ du capital social. ${ }^{22}$ Cette formule d'association mixte a pour effet d'assurer les capitaux publics nationaux à des capitaux privés.

Il ressort de l'article 2 de la loi de 1990 que le secteur parapublic sénégalais est composé de trois structures que sont les établissements publics industriels et commerciaux, les sociétés nationales et les sociétés anonymes à participation publique majoritaire. Toutefois, le législateur a choisi l'énumération en déterminant le contenu du secteur parapublic mais il ne définit pas l'entreprise publique compte tenu de la complexité de cette notion.

Afin d'expliquer la difficulté de définir précisément l'entreprise publique, il est opportun de citer l'analyse de Jean Rivero: « La famille du vocabulaire juridique compte deux catégories de membres. Les uns n'ont jamais appartenu au vocabulaire commun d'où la précision de leur définition, qui ne laisse place à aucune ambiguïté. D'autres sont en quelque sorte des enfants adoptifs. La langue du droit les a intégrés à partir de la langue courante où ils étaient jusque-là développés. Quels que soient les efforts faits pour leur donner la rigueur requise par leur entrée dans la langue du droit, ils gardent, de leur origine, un flou difficile à corriger totalement, et leur définition s'en ressentent $\gg{ }^{23}$ La notion d'entreprise publique appartient-elle aussi à ce deuxième groupe? Ce flou caractérise même l'ensemble du droit public économique pour certains auteurs comme Gérard FARJAT qui affirme « qu'en réalité le droit économique vit sans définition ${ }^{24}$ notamment après les échecs législatifs.

Il apparait clairement que la définition de l'entreprise publique n'est évidente. Il s'y ajoute que le régime juridique des entreprises du secteur parapublic est ambivalent. Pour les EPIC, même si les textes ne prévoient pas de dispositions particulières, la qualité de commerçant peut être déduite de la pratique. En effet, est commerçant ou bien a la qualité de commerçant celui fait des actes de commerce et remplit des conditions d'inscription au registre. En ce qui concerne les Sociétés Nationales, aux termes de l'article 4 de la loi de 1990 précitée, elles sont définies comme des sociétés par actions de droit privé. Elles sont régies par le droit commun des sociétés commerciales sous réserve des dispositions dérogatoires prévues par la loi. L'article 6 de la même loi traite des Sociétés Anonymes à Participation Publique Majoritaire et précise qu'elles sont régies par le code des obligations civiles et commerciales. Aux termes de l'article 7 de la même loi, leurs règles de formation, d'organisation et de fonctionnement sont conformes au droit commun des sociétés commer-

22 Les règles d'organisation et de fonctionnement sont conformes au droit commun des sociétés commerciales sauf exception prévu par la loi.

23 Delvolve, P., Droit public de l'économie, 1998, Dalloz..

24 Farjat, G., Pour un droit économique, PUF, 2004. 
ciales. Au total, les entreprises publiques sénégalaises ont la qualité de commerçant mais il y a lieu de préciser que malgré l'existence des dispositions spécifiques, la qualité de commerçant des entreprises publiques est évidente et résulte surtout de l'obligation d'inscription au registre du commerce ou de l'obligation de tenir des livres de commerce et de l'application des règles de la comptabilité commerciale, de la soumission aux obligations fiscales de commerçants. Pour l'essentiel, le principe de la commercialité est indiscutable même si l'on peut brandir quelques particularités liées à la mission de service public ou l'exécution ou la réalisation d'un intérêt général.

Les interventions des entreprises publiques susceptibles de soulever des problèmes au point de vue juridique se situent dans le domaine de la responsabilité extra contractuelle. Ces interventions sont en principe soumises à un régime de droit commun. Les marchés conclus avec les tiers obéissent à un régime juridique plus complexe selon qu'il s'agit de marchés de fournitures ou de marché de travaux publics. Concernant les contrats conclus avec les usagers, il convient de préciser qu'il s'agit en effet des contrats d'abonnement. Ces contrats sont souvent soumis au droit commun. Les litiges qu'ils peuvent éventuellement soulever relèvent de la compétence du juge judiciaire. Ce régime juridique résulte de la jurisprudence relative aux contrats conclus par les SPIC avec les usagers. ${ }^{25}$ Dans certains cas, le problème peut se poser de savoir si le différend porte sur les relations contractuelles du service public avec les usagers ou au contraire sur l'organisation du service public, ce qui peut entraîner la compétence du juge administratif. ${ }^{26}$

Il convient de signaler que le cas des contrats d'assurance souscrits par les entreprises en forme d'EPIC. Ces contrats sont également des contrats de droit privé, même lorsque sont évoqués à l'occasion d'un dommage de travaux publics. ${ }^{27}$ Cette décision est conforme à la jurisprudence constante du TC. ${ }^{28}$ Aussi, les contrats passés par les entreprises publiques avec leurs fournisseurs sont des conventions soumises au droit privé. Cependant ces contrats peuvent relever du droit administratif et par conséquent des juridictions administratives en cas de litige à la condition d'avoir été conclu par un établissement et de prévoir soit des clauses exorbitante de droit commun, soit l'association du fournisseur à l'exécution d'une mission de service public. ${ }^{29}$ De telles conditions sont rarement réunies, les fournisseurs se contentant d'opération de livraison qui ne les associent pas directement au fonctionnement du service public. En outre, les dommages causés aux usagers des entreprises publiques relèvent de la compétence du juge judiciaire. ${ }^{30} \mathrm{Il}$ importe peu que l'entreprise ait une forme juridique sociétaire ou d'établissement public et que le dommage soit causé ou non à l'occasion du fonctionnement ou de l'utilisation d'un ouvrage public ou d'un travail

25 Voir Durupty, M., Existe-il un critère de l'entreprise publique? Rev. Adm, 1984.

26 Eod. loc.

27 TA de Toulouse, 14 mars 1975, Caisse Assurance Maladie de la Haute Garonne.

28 TC, 26 nov. 1973, Société Assu. Cie AUP.

29 Mescheriakoff, A.-S., L'Autonomie des entreprises publiques, R.D.P, 1575.

30 Eod. loc. 
public. Le seul élément important à prendre en compte est la qualité d'usager des services rendus par l'entreprise. Concernant les rapports entre le tiers et l'entreprise publique, il faut distinguer deux (02) situations selon que le dommage est causé au tiers ou au contraire par le tiers à l'entreprise. En effet, les dommages causés aux tiers par les entreprises publiques sont les principes de droit commun sans qu'il soit nécessaire de tenir compte de la forme juridique de l'entreprise publique, ni du problème de savoir si elle gère une activité de service public. Les tribunaux judiciaires sont donc compétents pour apprécier les demandes de réparation effectuées par les victimes. Les dommages causés aux entreprises publiques par les tiers sont également réparés sur le fondement du droit privé. Il en va ainsi par exemple en cas d'accident du travail subis par les membres du personnel d'une entreprise publique. Une jurisprudence importante en matière de diffamation a confirmé que les préjudices en résultant, lorsque les entreprises publiques sont en cause, relevaient de la compétence du juge judiciaire pour leur réparation. ${ }^{31}$

L'application du droit public, même pour l'entreprise publique « la plus privée » est liée à l'appartenance de l'entreprise publique au secteur public. ${ }^{32}$ Tant qu'elle y reste ses rapports avec les pouvoirs publics sont régis par le droit public, notamment pour les mesures de nomination de leurs dirigeants et les mesures de contrôle de leur activité. Pour la faire sortir du secteur parapublic, ce sont les règles du droit public qu'il faut mettre en œuvre. La mission de service public de certaines d'entre elles conduit à voir des actes administratifs dans les règlements qu'elles prennent pour son organisation même si elles sont des personnes privées ${ }^{33}$ et à leur appliquer les lois du service public: continuité, égalité, adaptation constante. Malgré l'affirmation du caractère commercial de la gestion de certaines entreprises publiques, les principes de la gestion publique trouvent à s'appliquer tantôt au niveau des procédures et des organes de la comptabilité publique, tantôt au niveau du recouvrement des créances des entreprises publiques. Il convient en outre de souligner que toutes les entreprises publiques sont soumises au contrôle de la Cour des Comptes ayant reçu compétence pour l'exercice de ce type d'investigation. L'application des principes de la comptabilité publique et la présence de comptables publics relevant du contrôle de la Cour des Comptes caractérisent les entreprises publiques.

Aussi, critiquées, en raison de leur caractère parfois brusque et unilatéral, les interventions économiques des personnes publiques empruntent de façon croissante la voie du partenariat, substituant ainsi la concertation au commandement. En effet, le développement des partenariats s'exprime, d'abord, par une contractualisation croissante des rapports entre les personnes publiques et les personnes privées. Cette contractualisation résulte d'une tendance à l' " externalisation » des activités économiques d'intérêt général qui sont confiées de façon croissante aux personnes privées. Les partenariats contractuels se caractérisent par leur grande diversité, en fonction de l'imagination des parties et de la pluralité des finalités

31 Cass. Civile 15 novembre 1995, Cass et C/caisse régionale d'assurance vieillesse Alsace.

32 Delvolve, P., Droit public de l'économie, Dalloz, $1998 .$.

33 Epoux Barbier TC, 15 janvier 1968. 
poursuivies : baux emphytéotiques administratifs, conventions de subvention, délégations de services publics, contrats de partenariats publics-privé. Les partenariats publics-privés peuvent également avoir une dimension institutionnelle.

Le développement des partenariats concerne également les rapports entre les personnes publiques. Ces dernières sont, en effet, conduites à mutualiser leurs moyens pour mieux satisfaire les besoins des administrés. Toutefois, le recours des personnes publiques aux partenariats doit se faire dans le respect du principe d'égal accès à la commande publique, des libertés de circulation et de l'interdiction des discriminations fondées sur la nationalité. Il en résulte la soumission des personnes publiques à un droit de la mise en concurrence, plus ou moins contraignant selon la nature et les enjeux du partenariat, et dont le champ d'application s'avère parfois incertain. Dernier né dans le champ de la commande publique, le contrat de partenariat public-privé vient enrichir le droit des contrats administratifs. Partant de l'observation selon laquelle, l'Afrique a besoin de développer ses infrastructures et services de distribution dans les secteurs des transports, de l'énergie, de l'eau et des technologies nouvelles de communication pour promouvoir le développement économique et social, des organisations multilatérales et notamment la Banque mondiale, ont considéré que l'effet de construction de ces infrastructures doit être soutenu par l'implication des opérateurs privés. C'est la raison pour laquelle, le recours au partenariat public-privé ne cesse de se développer dans de nombreux secteurs clés de la commande publique et qui connaît un succès particulier ces derniers temps dans les pays d'Afrique. ${ }^{34}$ On peut soutenir à juste titre comme le montre un spécialiste, que l'installation de cette technique contractuelle se justifie aisément au cas où l'Etat devait se charger de contrôler l'exécution de certains travaux d'infrastructures qu'il n'a pas les moyens de financer. ${ }^{35}$ De ce point de vue, le partenariat public-privé est un contrat à long terme par lequel la puissance publique décide de confier à des entreprises généralement privées la mise en œuvre d'une mission d'intérêt général. Le contrat de partenariat n'est pas un marché public, le contrat de partenariat n'est pas une convention de délégation de service public. S'agissant de la définition légale et du domaine du contrat de partenariat au Sénégal, on constate que l'article 10 nouveau du code des Obligations de l'Administration définit le contrat de partenariat comme: le contrat par lequel une personne publique confie à un tiers, pour une période déterminée, une mission globale comprenant le financement et la réalisation, y compris la construction, la réhabilitation ou la transformation d'investissements matériels ou immatériels, ainsi que leur entretien.

A côté des entreprises du secteur parapublic au sens de la loi de 1990 précitée, nous constatons l'irruption des agences d'exécution dans le secteur parapublic qui constitue une autre évolution intéressante qui va influer sur le droit public économique sénégalais.

34 Essai de définition d'une nouvelle catégorie de contrat administratif, une approche comparée: Bénin, Burkina Faso, Cameroun, Gabon, Mali, Niger.

35 Ngaïde, M., La loi n²006-16 du 30 juin 2006 sur le renouveau du droit des contrats administratifs au Sénégal, article déjà. 


\section{B. L'irruption des agences d'exécution dans le secteur parapublic sénégalais}

Le secteur parapublic sénégalais connaît depuis peu une mutation profonde. Celle-ci est le fait de structures qui ont connu un développement fulgurant dans la conduite des politiques publiques $^{36}$ avec l'introduction des agences d'exécution qui fonctionnent suivant des mécanismes juridiques nouveaux. ${ }^{37}$ Expérimentée, non sans difficultés par l'Agence d'Exécution des Travaux d'Intérêt Public contre le sous-emploi (A.G.E.T.I.P.) en 1989, l'agenciation s'est généralisée dans le champ administratif et économique sénégalais. Presque tous les secteurs d'activités sont concernés. ${ }^{38}$ On en dénombre 30 à partir du décret $n^{\circ} 2006-267 \mathrm{du}$ 23 mars $2006^{39}$ et le recensement effectué en mai 2006, dans le cadre de la mission d'évaluation des agences commanditée par l'administration fait état de « plus de soixante agences dans l'environnement administratif $»{ }^{40}$ En effet, comme la plupart des pays en voie de développement, les exigences de la société moderne en matière de service public, de régulation du marché, de promotion d'un environnement plus favorable aux investissements, d'infrastructures si l'on connaît la faiblesse des Etats d'Afrique subsaharien, en l'occurrence le Sénégal, en matière de structures d'équipement collectif, ont caractérisé l'orientation des politiques publiques de ces dix dernières années. Cette nouvelle donne traduit la rapidité de l'évolution économique et sociale.

La constitution d'espaces économiques d'intégration telles que l'Union Economique et Monétaire Ouest Africain (UEMOA) exige des Etats africains l'adaptation de leurs législations mais aussi de leur environnement administratif. En effet, l'Etat sénégalais a procédé à la mise en place d'institutions chargées d'activités de prestations de services qui se traduisent dans les faits par la création d'agences autonomes. Il s'agit pour les pouvoirs publics de déléguer la gestion de certaines missions à des organismes dotés d'une certaine indépendance d'action. Seule l'indépendance les distingue de l'administration traditionnelle. Leur création est motivée par des soucis d'efficacité de l'action publique et la recherche d'une certaine souplesse dans la gestion et d'une certaine flexibilité, impossible dans une administration classique. ${ }^{41}$ Ainsi, l'Etat sénégalais va créer à côté des structures ministé-

36 Chevallier, Jacques, Science administrative, PUF, 1994, p. 410.

37 Abate, Bernard, La nouvelle gestion publique, L.G.D.J., Paris, 2000, p. 91.

38 Jusqu'en 2000, ils n'étaient que cinq, puis ce fut la prolifération: quatre nouvelles en 2000, six, en 2001, cinq l'année suivante. En 2004, sept nouvelles voient le jour, auxquelles s'ajoutent cinq autres en 2005.

39 Décret $\mathrm{n}^{\circ} 2006-267$ du 23 mars 2006 portant répartition des services de 1'Etat et du contrôle des établissements publics, des sociétés nationales et des sociétés à participation publique entre la présidence de la République, la primature et les ministères.

40 Rapport Final de la Mission d'évaluation des agences, Délégation au Management Public, 20 juillet 2006, Dakar, p. 55..

41 C'est ainsi que le gouvernement sénégalais a procède par externalisation de services administratifs, les érigeant en agences comme ce fut le cas dans le domaine de la statistique avec l'Agence Nationale de la Statistique et de la Démographie du Sénégal (ANSD) qui reprend les activités statistiques de la Direction de la Prévision et de la Statistique (DPS). 
rielles classiques, des agences autonomes chargées de la mise en œuvre de politiques publiques. $^{42}$

La loi d'orientation 2009-20 du 04 mai 2009 portant sur les agences d'exécution vise à harmoniser et à encadrer la pluralité et la diversité des modes de création, d'organisation et de fonctionnement des agences, par la définition de principes directeurs communs. A ce titre, il définit de manière précise le statut, l'objet, les conditions de création et de dissolution des agences. Dans cette perspective, il est retenu de doter les agences de la personnalité morale de droit public avec des tâches et des missions précises. La même loi prévoit aussi que toute proposition de création d'une agence devra désormais être justifiée par une analyse d'impact.

Selon l'article premier de la loi 2009-20 du 04 mai 2009 portant loi d'orientation sur les agences d'exécution définit l'agence d'exécution comme une entité administrative autonome, investie d'une mission de service public. Elle est une personne morale de droit public dotée d'un patrimoine et de moyens de gestion propres.

L'agence est créée par décret pour une durée déterminée ou indéterminée, sur proposition du chef de l'Administration de tutelle technique, en vue de remplir des tâches de nature technique, scientifique ou de gestion bien spécifique.

En réalité, la plupart des agences d'exécution sénégalaises sont créées par décret mais la pratique des agences au Sénégal est complexe. Tel est le cas de l'APIX, qui créée par décret n`2000-562 du 10 juillet 2000 n'était qu'une simple structure autonome. ${ }^{43}$ Elle peut être un établissement public, comme l'Agence de Presse Sénégalaise (APS), qui au regard de l'ordonnance n59.054 du 2 avril 1959, modifiée par la loi n72-72 du 26 juillet 1972 " est un établissement public industriel et commercial ». L'agence d'exécution n'est cependant pas obligatoirement un organisme public. Elle peut être une personne privée auquel cas, l'agence peut être une association, un projet ou alors une entreprise publique à forme sociétale. A cet effet, les mutations du statut de l'APIX nous en donne une bonne illustration. En effet, celle-ci a ouvert son capital au privé, en devenant une société anonyme à participation publique majoritaire après avoir été une société entièrement publique, au travers de la loi n²007-13 du 6 février 2007. En conséquence, il leur est applicable le régime juridique attaché à ces statuts juridiques.

En réalité, à y regarder de près, les agences d'exécution fonctionnent comme des entreprises publiques. Ainsi, elles sont placées sous la tutelle technique de son administration de rattachement et sous la tutelle financière du Ministre chargé des finances. Aussi, chaque agence, comme l'entreprise du secteur parapublic, est soumise à un

42 Ce qui est recherché dans ce type d'agence, c'est l'efficacité, la performance dans les résultats, résultats dont dépend, en principe leur pérennité. On range dans cette catégorie la majorité des agences sénégalaises. Il en est ainsi de l'AGETIP, de l'Agence Autonome des Travaux Routiers (AATR), de l'Agence de la Case des Tout-petits etc. Contrairement aux agences de régulation, leur développement a été le plus marqué.

43 Article premier du décret $n^{\circ} 2000-562$ du 10 juillet 2000 portant création et fixant les règles d'organisation et de fonctionnement de l'APIX, J.O.R.S. du 15 juillet 2000. 
contrat de performance. Sur un plan organique, les agences disposent comme les entreprises publiques d'un organe exécutif en l'occurrence un directeur général et d'un organe de surveillance. Sur le plan fonctionnel, les agences d'exécution comme les entreprises du secteur parapublic sont en principe, soumises au Code des Marchés. ${ }^{44}$ A cela s'ajoute le fait que le statut du personnel des agences d'exécution est en principe un statut de droit privé, au même titre que celui des entreprises publiques. Ainsi l'article 19 du décret du 3 août 2000 relatif à l'AATR stipule : «Le personnel de l'Agence, ainsi que le secrétaire exécutif et ses collaborateurs, sont soumis à la réglementation du travail du secteur privé $»{ }^{45}$ De même la comptabilité des agences est une comptabilité privée comme l'atteste l'article Article 12 de la loi 2009-20 du 4 mai 2009 qui stipule que selon son mode de gestion, l'agence peut appliquer les règles de la comptabilité privée ou celles de la comptabilité publique. ${ }^{46}$ Le référentiel comptable applicable ainsi que les modalités particulières de gestion financière et comptable sont définis par le décret de création de l'agence. Toutefois, le recouvrement des recettes et le règlement des dépenses de l'agence sont assurés par un agent comptable nommé par arrêté du Ministre chargé des Finances.

Aussi, chaque agence d'exécution est soumise à un contrat de performance à l'instar du contrat-plan. Par ailleurs, les agences sont soumises au contrôle de la Commission de Vérification et de Contrôle des Comptes des Entreprises publiques (CVCCEP) ${ }^{47}$ En effet, selon l'article 13 de la loi d'orientation sur les agences d'exécution, sans préjudice de tout autre contrôle légal ou règlementaire, les comptes des agences qui utilisent les règles de la comptabilité publique sont soumis à un audit externe et les comptes

44 Code des marchés publics, issu du décret n²002-550 du 30 mai 2002 (toutefois ce décret a été remplacé par le décret n²007-545 du 25 avril 2007 portant Code des Marchés Publics).

45 Décret n²000-686 du 3 août 2000 relatif à la création, à l'organisation et au fonctionnement de l'Agence Autonome des Travaux Routiers et du Conseil des Routes.

46 Ainsi selon11 du décret de 2000 portant création et fixant les règles d'organisation et de fonctionnement de l'APIX. Il dispose que « La comptabilité de l'Agence est tenue suivant les règles et les principes de la comptabilité privée ». De même, l'article 12 du décret du 26 mai 2005 relatif à l'Agence de Mise en œuvre de la Plate-forme du Millénaire de Diamniadio (AMPMD), pose que «La comptabilité de l'Agence est tenue suivant les règles et principes de la comptabilité privée ». Le décret n²000-686 du 3 août 2000 relatif à l'AATR, est plus explicite; il stipule en son article 20 : «L'agence tient une comptabilité et doit produire des états financiers annuels conformément au système comptable en vigueur pour les entreprises commerciales ». Les exemples sont nombreux, l'Agence Nationale de l'Organisation de la Conférence Islamique (ANOCI), ainsi que l'Agence Sénégalaise de Promotion des Exportations (ASEPEX) sont soumises, en ce qui concerne leur régime comptable, à des règles plus ou moins éloignées de celles, très strictes, de la comptabilité publique. Il n'est pas dit que toutes les agences d'exécution sont plus ou moins proches des entreprises du secteur parapublic, mais que beaucoup d'entre elles, qui généralement correspondent à ce qu'on pourrait appeler les « grandes » agences de l'Etat, nous font penser à des entreprises publiques.

47 Ainsi, l'article 12 du décret n²001-211 du 13 mars 2001 portant création et fixant les règles d'organisation et de fonctionnement de l'Agence Sénégalaise pour l'Innovation Technologique (ASIT), dispose : « L'agence est, en outre, soumise au contrôle de la Commission de Vérification et de Contrôle des Comptes des Entreprise publiques ». 
utilisant le système comptable ouest africain pour enregistrer leurs opérations sont contrôlés par un commissaire aux comptes. Le commissaire aux comptes ou l'auditeur privé est choisi par le conseil de surveillance de l'agence qui fixe ses honoraires. Les agences sont tenues de produire périodiquement des rapports relatifs à l'exécution de leur budget et de leur trésorerie qu'elles adressent à la tutelle technique et à la tutelle financière, sans préjudice des états financiers et des rapports annuels.

L'évolution de certaines agences vers le statut de l'entreprise du secteur parapublic est même illustrative. ${ }^{48}$ Dans cette perspective, la Cour des comptes ne fait pas de distinction entre Entreprises publique et Agence d'exécution. ${ }^{49} \mathrm{Au}$ surplus, les justifications évoquées par le législateur dans la création des agences d'exécution attestent de manière claire que celles-ci interviennent dans le secteur parapublic. Aux termes de 1'article 2 de la loi de 2009, la création d'une agence doit être justifiée par des nécessités fonctionnelles relevant d'une volonté politique de donner plus d'impulsion, d'autorité et d'autonomie à un ensemble d'activités nouvelles ou insuffisamment prises en charge par les services administratifs et du souci de rendre un service de qualité aux usagers en apportant des solutions appropriées fondées sur la proximité, la participation et l'adaptabilité, que des services centraux ne peuvent pas assurer mais aussi de la nécessité de rendre l'Administration plus attentive à la notion de performance et de résultats.

\section{Une régulation économique revalorisée}

Pour asseoir une économie de marché, le Sénégal a fait le choix du désengagement de l'Etat dans l'administration de l'économie et accordant une priorité au secteur privé. Dans cette perspective, le Sénégal mis en place un système de régulation spécifique. En effet, c'est surtout dans sa dimension institutionnelle que l'on mesure toute l'ampleur du bouleversement qu'a entrainé la régulation surtout dans les secteurs d'infrastructures ${ }^{50}$ à titre d'illustration. La raison évoquée réside dans l'ouverture à la concurrence d'un secteur naguère monopolistique. Dans un sens assez littéral, le terme de régulation est désormais régulièrement utilisé dans la sphère économique et des politiques publiques pour désigner tout ce qui est

48 L'exemple de l'APIX, à cet égard est révélateur. En effet ce n'est que très récemment, et après de longues tergiversations des pouvoirs publics, qu'elle s'est vue octroyée le statut de société anonyme à participation publique majoritaire et partant celui d'entreprise du secteur parapublic au travers de la loi n 13-2007 du 6 février 2007, alors qu'elle n'était, au départ, qu’une simple structure autonome.

49 Voir les rapports publics de la Cour des Comptes 2006, 2007, 2008, 2009 et 2010 (www.courdescomptes.sn).

50 Au Sénégal, le législateur a procédé à une définition de la régulation économique au travers de la loi $\mathrm{n}^{\circ}$ 2002-23 du 4 septembre 2002 portant cadre de régulation pour les entreprises concessionnaires de services publics. Dans son exposé des motifs, il estime qu': " A l'instar de la pratique internationale en la matière, la régulation visée ici renvoie aux mécanismes de correction des défaillances du marché ». Autrement dit, il s'agit de l'ensemble des procédés et mécanismes par lesquels, les pouvoirs publics tentent de trouver et de maintenir un équilibre optimum du marché. . 
fait pour apporter par une action appropriée à la marge, une stabilité satisfaisante à un système économique ou même social. Il s'agit donc de la recherche d'un certain équilibre effectuée à travers la mise en œuvre de divers mécanismes. Le Pr. Frison-Roche décline trois sens du mot régulation à savoir l'organisation de l'exercice du pouvoir par son titulaire, ensuite le rééquilibrage de rapports de force et enfin l'organisation permanente de secteurs économiques qui ne sont pas laissés au seul principe de concurrence. ${ }^{51} \mathrm{Il}$ apparaît que c'est ce dernier sens de la régulation qui est plus ou moins généralement admise.

La régulation renverrait du point de vue du Pr. Frison-Roche à « un appareillage de secteurs construits sur un principe de concurrence en équilibre avec d'autres impératifs ». ${ }^{52}$ Dans une approche matérielle, la régulation économique consisterait à organiser la libéralisation de services en réseaux autrefois fournis par des entreprises (généralement publiques) dans le cadre de monopoles réglementés par l'Etat. La coexistence aujourd'hui dans un même secteur de missions d'intérêt général assurées par un seul opérateur et d'activités ouvertes à la concurrence requiert une action publique spécifique qui parvienne à concilier ces deux principes apparemment contradictoires. A côté d'une conception purement matérielle, l'on pourrait avoir une vision organique de la notion de régulation économique. Dans cette perspective celle-ci se confondrait avec l'action de certains organismes communément appelés institutions, organes, commissions bref pour être plus précis autorités de régulation. La récente vogue que connaissent ces institutions surtout dans le cadre sénégalais, témoignent de l'intérêt que suscitent la régulation économique dans nos jeunes Etats.

$\mathrm{Au}$ regard des textes qui régissent la régulation économique, il est important d'aborder les différentes innovations introduites en droit positif sénégalais en matière de régulation économique (A) avant d'apprécier ce nouveau cadre à travers l'encadrement juridique de la régulation économique $(\mathrm{B})$.

\section{A. Les innovations introduites par le positif en matière de régulation économique}

C'est plus précisément l'article 2 de la loi de 2002 qui, en droit positif sénégalais, confère explicitement la qualité d'autorité administrative indépendante (AAI) aux organes de régulation. En effet, selon l'article 2 précité : « (...) L'institution de régulation est une autorité administrative indépendante... ». La nature d'AAI qui est affectée aux institutions de régulation, les situe par conséquent hors de la hiérarchie des structures administratives traditionnelles. Dans cette perspective, l'Agence de Régulation des Télécommunications et des Postes (ARTP) au Sénégal ainsi que le Conseil de Régulation du Secteur de l'Electricité (CRSE), de même que l'Autorité de Régulation des Marchés Publics (ARMP) ${ }^{53}$ issue de la

51 Frison-Roche, Marie-Anne, Définition du droit de la régulation économique, D. 2004, chronique.

52 Eod. Loc.

53 Dans le cadre de l'assainissement des finances publiques en termes de transparence et d'équité, l'UEMOA impose aux Etats membres la création d'organes de régulation. Cette création d'organes de régulation dans l'espace sous régional est pour la plupart une exigence de l'Union surtout en matière de marchés publics. C'est le cas en effet de l'Autorité de régulation des Marchés publics 
loi $\mathrm{n}^{\circ}$ 2006-16 du 30 juin 2006 modifiant le Code des Obligations de l'Administration, peuvent être considérées comme des AAI. L'octroi du statut d'AAI permet donc de conférer aux organes de régulation la distance nécessaire pour rendre efficace leur mission de régulation. En réalité, l'indépendance des autorités ne peut être effective qu'avec l'octroi de moyens matériels, financiers accompagnés d'une autonomie de gestion. ${ }^{54}$ Le législateur va plus loin avec l'octroi de la personnalité morale aux institutions de régulation. ${ }^{55}$ Sur cette question, la doctrine est divisée car l'attribution de la personnalité morale aux organes de régulation peut paraître paradoxale en ce que l'AAI, selon certains auteurs comme le professeur Jacques CHEVALLIER, est dépourvue de la personnalité juridique parce qu'elle représente juridiquement l'Etat. ${ }^{56}$ Par ailleurs, la personnalité morale peut ainsi être appréhendée comme un renfort à l'indépendance de ces organes de régulation et permet de faire de ces derniers des personnes responsables capables d'ester en justice. Le législateur sénégalais a opté pour cette deuxième solution.

Sous un autre registre, l'autorité de régulation met en œuvre les règles permettant l'établissement et le maintien d'un marché concurrentiel. A ce titre, l'organe de régulation dispose d'un pouvoir important comme le « pouvoir d'enquête " prévu à l'article 6 de la loi de 2002. Dans ce contexte, il est donc essentiel que les autorités administratives indépendantes soient dotées des réels pouvoirs juridiques. Cependant les interventions du législateur ne sont pas allées dans le sens de la cohérence dégagée par la loi de 2002, et à cet effet, l'exemple de l'ARTP est très illustratif. ${ }^{57}$ Son statut d'établissement public fausse la logique d'indépendance que le législateur voulait assigner à l'autorité de régulation, en ce que

au Sénégal. L'ARMP est tout d'abord une autorité administrative indépendante dotée de l'autonomie financière et de gestion. Il est aussi une autorité sectorielle de régulation spécialisée dans le domaine des marchés publics. Elle est instituée par la loi No 2006 -16 du 30 juin 2006 modifiant le COA à son article 30. Ce projet distingue l'ARMP de l'autorité administrative chargée de contrôle a priori de la passation des marchés publics dont la composition, la compétence et le mode de fonctionnement seront précisés par décret. Elle connait le recours non juridictionnel en matière des marchés publics. La mission de l'ARMP est selon l'article 2 du décret 2007-546 du 25 Avril 2007 portant organisation et fonctionnement de l'ARMP « d'assurer la régulation du système de passation des marchés publics et des délégations de services publics et le règlement non juridictionnel des litiges assuré par le comité de règlement des différends établi auprès de l'ARMP ».

54 L'autonomie financière et de gestion que leur octroie la loi de 2002 est une conséquence logique qui résulte de la qualité d'AAI des organes de régulation.

55 En effet les organes de régulation des secteurs d'infrastructures sénégalais aux termes de l'article 10 de la loi de 2002 « ... disposent de la personnalité juridique ».

56 Chevallier, Jacques, Réflexions sur l'institution des AAI, JCP 1986. I. 3254.

57 Aux termes de l'article 4 de la loi n²006-02 du 4 janvier 2006 modifiant la loi n²001-15 du 27 décembre 2001 portant code des télécommunications : « L'ARTP est un établissement public dotée de la personnalité juridique et de l'autonomie financière ». L'ARTP présente aussi bien sur le plan organisationnel que fonctionnel, les caractéristiques d'un établissement public, mais tel ne devrait pas être le cas. 
l'établissement public est soumis à la tutelle. ${ }^{58}$ Ainsi la problématique de l'ancrage institutionnel des régulateurs des services publics est donc une question essentielle dans la perspective des missions de régulations. ${ }^{59}$ Pour asseoir une quelconque autorité, les instances de régulation doivent d'abord être indépendantes. ${ }^{60}$ Cette question est surtout envisagée sous l'angle de l'indépendance des membres des instances de régulation puisqu'en réalité c'est eux qui prennent les véritables décisions. ${ }^{61}$ Cette indépendance est donc essentielle et doit se mesurer au plan organique et fonctionnel. Sur un plan organique, la loi de 2002 sur les entreprises concessionnaires de service public dispose en son article 11 que les membres des agences de régulation seront nommés sur une base non partisane et suivant des qualifications reconnues. Durant leur mandat, les personnels cadres des institutions de régulation ne peuvent être révoqués, sous réserve des dispositions relatives à l'éthique et à la bonne gouvernance. L'article 8 du décret du 21 avril 1998 relatif à la Commission de Régulation du Secteur de l'Electricité (CRSE), prévoit que les membres de la Commission de Régulation du Secteur de l'Electricité ne peuvent être poursuivis, recherchés, arrêtés, détenus ou jugés à l'occasion des opinions et décisions exprimées, ou des votes émis ou des actes commis dans l'exercice de leurs fonctions. ${ }^{62}$ Des dispositions plus ou moins semblables peuvent être trouvées dans les textes relatifs à l'organisation et au fonctionnement des institutions de régulation. Sur un plan fonctionnel, les agences de régulation ${ }^{63}$ ne sont pas en théorie soumises à la tutelle, malgré le fait qu'elles agissent pour le compte de l'Etat.

Par ailleurs, les agences de régulation disposent d'un budget autonome qu'elles adoptent, arrêtent de manière définitive et utilisent en principe librement. A travers le budget, s'affiche une volonté politique de mettre plus ou moins les agences dans les conditions optimales d'exercice de leurs missions.

58 Par ailleurs, dans le fonctionnement de la régulation, les ministères de tutelle jouent un rôle non négligeable. Selon l'article 3 de la loi de 2002 précitée: « Les ministères de tutelles des secteurs régulés sont chargés de la définition des politiques sectorielles pour le compte du gouvernement. Les ministères veillent à la promotion du développement efficace du secteur conformément aux engagements souscrits avec les différents opérateurs et acteurs évoluant dans ledit secteur ».

59 En effet, l'article 42 du Code des télécommunications dispose : « Il est créé sous l'autorité du Président de la République l'agence.... ». Cette disposition pose la problématique de l'indépendance des instances de régulation qui constitue un préalable indispensable.

60 La mission de régulation exige plus qu'une simple autonomie généralement octroyée à certaines administrations lorsqu'on veut leur donner une certaine marge de manœuvre.

61 C'est ainsi que Marie-José Guédon précise que :"l'indépendance de l'autorité implique d'abord l'absence de toute tutelle ou pouvoir hiérarchique à son égard de la part du pouvoir exécutif. Une AAI ne reçoit ni ordre, ni instruction du Gouvernement" op. cit.

62 Décret n ${ }^{\circ} 998-333$ du 21 Avril 1998 portant organisation et fonctionnement de la Commission de Régulation du Secteur de l'Electricité.

63 Article 5 du décret N²007-546 du 25-04-2007 portant organisation et fonctionnement de l'Autorité de régulation des marchés publics (ARMP). 


\section{B. L'encadrement juridique de la régulation économique}

Le caractère distinctif des ordres régulations provient de ce que les organes de nature administrative qu'ils font naître remplissent un rôle de nature juridictionnelle qui bouscule les idées reçues de Montesquieu sur la distinction des pouvoirs. ${ }^{64}$ C'est pourquoi, les A.A.I. posent un problème de qualification substantielle non plus au regard du droit public mais en ce qui concerne le rôle extraréglementaire et administratif qui est le leur. Le mode d'action principal des autorités régulatrices n'est pas de réglementer l'économie mais de réguler le fonctionnement de tel ou tel secteur d'activités économiques. Pour ce faire, les autorités de régulation sont chargées d'instruire le procès des entreprises et/ou de tout agent économique qui entrent en infraction tant avec les règles législatives et/ou réglementaires qu'elles sont chargées de faire respecter qu'avec les normes qu'elles édictent dans l'exercice de leurs délégations de compétences régaliennes. À cet égard, elles disposent de la jurisdictio, c'est-à-dire du pouvoir de « dire le droit » analogue à celui qui est dévolu à toute juridiction, que celle-ci soit étatique ou qu'elle soit arbitrale. On sait que, dans la pratique, la jurisdictio emporte une prérogative d'interprétation des silences, des lacunes ou des ambiguïtés de la loi qui donne aux juges une fonction sociétale créatrice de Droit. ${ }^{65}$

Toutefois, les décisions des autorités de régulation au Sénégal peuvent être contrôlées par le juge. En effet, le rôle du juge dans la régulation économique, plus précisément dans la régulation des services en réseaux met en relief les pouvoirs des autorités de régulation, qualifiés de quasi-juridictionnels par une bonne partie de la doctrine. ${ }^{66}$ En effet, selon JeanLouis Autin « Il n'est pas contestable que lorsque les régulateurs règlent des litiges ou sanctionnent ceux qui violent les règles encadrant l'activité dans certains secteurs économiques, ils empiètent sur la sphère de compétence du juge même s'il est de moins en moins discuté de l'opportunité de leur intervention dans ce domaine $»{ }^{67}$ Au Sénégal, les pouvoirs de sanction des institutions de régulation sont expressément prévus à l'article 7 de la loi de 2002, qui accorde que les institutions de régulation disposent, dans le cadre de la mise en œuvre des responsabilités d'un pouvoir de sanction des manquements des entreprises des secteurs régulés. ${ }^{68}$ En outre, l'article 8 de la loi de 2002 permet d'attaquer les décisions de l'autorité de régulation devant la juridiction administrative en l'occurrence la chambre administration de la Cour suprême ${ }^{69}$ qui stipule que les décisions prises par les institutions de

64 Champaud, C., Régulation et Droit Économique, Ride, 2002.

65 Eod. loc.

66 Autin, J.-L., Du juge administratif aux autorités administratives indépendantes: un autre mode de régulation, RDP 1988, p. 1214.

67 Ibid.

68 En effet, les décisions de ces derniers sont, la plupart du temps, des décisions administratives qui, n'ayant donc pas le caractère d' " autorité de la chose jugée » mais plutôt d' "autorité de la chose décidée », ne bénéficient pas de l'ensemble des dispositions coercitives permettant l'exécution des décisions de justice, même si ce sont des décisions par nature exécutoires comme toute décision administrative.

69 Loi organique 2008-35 sur la Cour suprême du 8 août 2008. 
régulation peuvent faire l'objet de recours devant les instances compétentes. Le recours n'est pas suspensif. Toutefois le sursis à exécution de la décision peut être ordonné par la cour suprême si celle-ci est susceptible d'entraîner des conséquences manifestement excessives ou s'il est intervenu postérieurement à sa notification des faits nouveaux d'une exceptionnelle gravité. ${ }^{70}$

Le rôle du juge dans le jeu de la régulation a pour mission première le contrôle de l'action des régulateurs, ce qui légitime, dans une certaine mesure celle-ci. ${ }^{71}$ Dans cette perspective, on peut à juste titre appréhender sa mission comme une fonction de régulation. ${ }^{72} \mathrm{Il}$ reste qu'en dernier analyse c'est lui le garant ultime de la légalité, et en dernier recours c'est lui qui a le dernier mot mais cette nouvelle dimension donnée à l'activité juridictionnelle amène les juges à sortir de leurs rôles traditionnels et de leurs modes habituels de raisonnement puisque cette fonction de régulation suppose non seulement d'avoir en permanence à l'esprit l'intérêt général, mais également très souvent de faire œuvre de création et d'innovation. Il s'agit là d'une mutation profonde du monde judiciaire dont certains magistrats ont parfaitement saisi la dimension. ${ }^{73}$

Pour ce qui est du contentieux de la légalité, le juge utilise les mêmes techniques applicables en matière du recours en annulation. Pour être légal, l'acte administratif litigieux en matière économique doit remplir un certain nombre de conditions. Les conditions de légalité des actes administratifs en matière économique sont les mêmes que celles du recours pour excès de pouvoir. Toutes ces conditions sont généralement regroupées sous deux rubriques. La première est relative à la légalité externe et concerne les conditions formelles de la légalité de l'acte. La seconde ayant trait à la légalité interne concerne les conditions matérielles de l'acte. Pour ce qui est des conditions de la légalité externe, elles portent sur deux éléments de l'acte administratif en matière économique, d'une part sur son auteur, d'autre part sur sa procédure d'élaboration. La règle c'est que l'auteur de l'acte administratif contesté doit être compétent sous peine d'illégalité de son acte. Il s'agit en effet, de la première condition de légalité de l'acte administratif car la compétence est un moyen d'ordre public. Le vice de forme qui correspond généralement à une omission ou à l'accomplissement incomplet des formalités de l'acte administratif contesté.

70 L'article 39 du Code des télécommunications dispose que les décisions de l'ART portant sanction peuvent faire l'objet d'un recours en annulation ou d'une demande de sursis à exécution devant la Cour suprême. Le recours n'est pas suspensif. Les mesures conservatoires prises par l'ART peuvent, après leur notification, faire l'objet d'un recours en annulation devant la Cour suprême. L'intervention du juge vient donc en complément de l'action coercitive du régulateur.

71 Il dénote aussi une autre réalité, à savoir que plus qu'un simple organe de contrôle des instances de régulation, le juge en vérifiant a posteriori les décisions des régulateurs fait indirectement œuvre de régulation.

72 Si son manque de spécialisation, son éloignement par rapport aux réalités qu'il doit saisir ainsi que la lenteur de ses décisions, de même que la rigidité de la règle de droit qu'il entend appliquer à des faits par nature évolutifs sont autant de facteurs qui tendent à le reléguer au second plan au profit $\mathrm{du}$ régulateur.

73 Ibid. 
La seconde condition que le juge administratif sénégalais doit vérifier concerne la légalité interne de l'acte contesté à partir de trois éléments de l'acte qui sont l'objet, ${ }^{74}$ les motifs $^{75}$ et le but. ${ }^{76}$

Toutefois, en matière économique le juge se montre prudent dans le contrôle qu'il peut exercer compte tenu de la technicité ou de la spécialisation auxquelles le champ d'application de la réglementation économique renvoie souvent. Malgré ces difficultés, le juge porte une attention particulière à la légalité de l'action économique en ayant recours à l'erreur manifeste d'appréciation ou encore au contrôle de proportionnalité. L'intervention du juge dans ce domaine de l'économie a permis une avancée importante de l'ensemble du contentieux administratif, particulièrement en ce qui concerne la motivation des actes de l'administration. ${ }^{77}$

74 L'objet de l'acte administratif correspond à son contenu. Pour être légal, le contenu de l'acte ne doit pas violer celui de l'acte qui lui est supérieur. C.E. du Sénégal, 24 novembre 1993, Babacar Ngom et employés de la SIPAO; bul. C.E. $n^{\circ} 15$.

75 Les motifs sont les considérations de fait ou de droit au vu desquelles, l'autorité administrative compétente prend un acte administratif. C.E. 6 février 1974, Cissé, Abdourahmane, Annales Africaines 1975, p.65; GAJA T1, p.171. C.E. 26 janvier 1994, Abdoul Aziz Sow c/ Ordre national des experts et évaluateurs agréés du Sénégal; Bul. № 29. C.S. 8 juin 1968, Baïla Haïmouth Sow; GAJA T1. p.123; C.E. 26 janvier 1994, Alla Ngom et autres; Bul. N 23.Pour être légales, les considérations de fait à la base de l'acte administratif unilatéral doivent non seulement être établies, mais aussi juridiquement bien qualifiées. C.E. français 14 janvier 1916 Camino; GAJA n ${ }^{\circ}$ 33. C.S. 23 mars 1966, Babacar Lô et Abdou Salam Diallo; GDJAS. T1, P.285; C.E. 24 avril 1996, Souleye Badiane; Bul. CE $n^{\circ} 55$. Les faits qui servent de fondement à l'acte administratif doivent correspondre à ceux pour lesquels l'autorité administrative a reçu compétence. C.E. français 4 avril 1914, Gomel, GAJA n ${ }^{\circ}$ 32. C.E. français 20 mars 1968, Société du Lotissement de la plage de Pampelonne, R.D.P., 1969, p.319; AJDA 1968, p.335. C.E. 28 mai 1971, Ville Nouvelle-Est, GAJA nº 103.

76 Le but de l'acte administratif est défini en fonction de l'intention de son auteur, c'est-à-dire des mobiles subjectifs qui le sous-tendent. C.E. 26 novembre 1975, Parizet; GAJA n ${ }^{\circ}$ 4. C.E. 24 juin 1960, Société FRAMPAR, GAJA n 94. C.S. 6 juin 1973, Dame Yaye Katy Dieng; Annales Africaines 1974, p.65; GAJAS, T1. p.145.

77 CE 26 janvier 1968 « société Maison Genestal » l'administration avait refusé un agrément fiscal normalement prévu par le code général des impôts pour les entreprises réalisant des opérations de décentralisation industrielles au motif (incomplet) que la société requérante ne présentait pas des avantages économiques suffisants. CE 27 novembre 1970 Agence maritime Marseille-Frêt: le juge impose la motivation des décisions prises par un organisme collégial statuant en matière économique. CE 13 novembre 1974, sieur Guizelin, obligation de motivation des décisions prises par un organisme professionnel en égard à sa nature, à sa composition et à ses attributions (commission nationale). Enfin, le juge judiciaire gère aussi un aspect essentiel du contentieux économique. 
Dans l'étape actuelle du droit public économique sénégalais, le juge administratif n'a pas encore développé une jurisprudence abondante. ${ }^{78}$ Toutefois, nous pouvons relever quelques décisions importantes. ${ }^{79}$

\section{Conclusion}

Il ressort de l'analyse que l'interventionnisme économique au Sénégal a connu des mutations certaines. Pour autant, les pouvoirs publics sénégalais n'ont pas renoncé à toute forme d'intervention sur le marché. Le besoin de services publics marchands de qualité, à des tarifs accessibles, tant au niveau national que local, est toujours réel et régulièrement revendiqué. En outre, les situations de crise ont mis en évidence la nécessité constante non seulement d'une nouvelle forme d'intervention dans le champ économique sénégalais mais aussi d'une revalorisation de la régulation économique.

78 En effet, certaines contrariétés pouvant donner matière à procès n'arrive souvent pas devant le juge du fait des nombreux mécanismes de règlements souples des litiges aménagés par les textes. Certaines contestations sont réglées au niveau de l'organe régulateur ou par le biais de l'arbitrage.

79 Dans un arrêt pris en son audience du 10 juin 2008, la juridiction administrative a rejeté la demande d'un consortium dont le dossier n'avait pas été pris en compte par l'autorité de régulation des télécommunications en l'occurrence l'ARTP. Une affaire qui a été porté au contentieux et qui a failli faire perdre à la société Sudan Telecom Company Ltd (Sudatel) l'attribution de la seconde licence de téléphonie globale au Sénégal. Le Conseil d'Etat a débouté le Consortium Expanet Sunu Télécom Joint Loyal Sud Groupe Télécommunication et confirmé la régularité du processus d'attribution, conduit par l'ARTP. Le Conseil d'Etat a rejeté comme mal fondée la demande en annulation pour refus de réception par l'ARTP de la soumission du Consortium Expanet Sunu Télécom ainsi que sa demande subséquente en annulation de l'adjudication de la licence au groupe Sudatel laquelle, selon la haute juridiction administrative, manque également de fondement. Dans un autre Arrêt n ${ }^{\circ} 10$ du 25 septembre 2008 (État du Sénégal c/ - Autorité de Régulation des Marchés Publics (ARMP) - Comité de Règlement des Différends de l'ARMP - Agence Autonome des Travaux Routiers (AATR)) :Il y a lieu, par application des dispositions des alinéas 1er et 2 de l'article 36 de la loi organique n ${ }^{\circ}$ 96-30 du 21 octobre 1996 sur le Conseil d'État modifiée par les lois organiques $\mathrm{n}^{\circ}$ 99-70 et $\mathrm{n}^{\circ}$ 99-72 du 17 février 1999, d'ordonner le sursis à l'exécution de la décision du comité de règlement des différends de l'Autorité de Régulation des Marchés Publics qui a retenu, d'une part, que les dispositions de l'article 37 paragraphe 3 du code des marchés publics, concernant le représentant du contrôleur financier dans les commissions des marchés, sont contraires aux dispositions des articles 13 et 14 de la directive $n^{\circ}$ 5/2005/CM/UEMOA et, d'autre part, que le contrôleur financier ne peut siéger dans la Commission des Marchés de l'Agence Autonome des Travaux Routiers comme membre. En effet, le moyen articulé contre cette décision et tiré de la violation, en premier lieu, de l'article ler de la loi organique sur le Conseil d'État et des articles 18 à 23 du décret $n^{\circ}$ 2007-546 du 25 avril 2007 portant organisation et fonctionnement de l'Autorité de Régulation des Marchés Publics et, en second lieu, des articles 37 al 3 du code des marchés publics, 13 et 14 de la directive $n^{\circ}$ 04/2005 de l'UEMOA, paraît sérieux en l'état actuel de l'instruction et le préjudice encouru par le requérant, en cas d'exécution de la décision attaquée, serait difficilement réparable pour le contrôleur financier qui ne pourrait plus jouer sa mission de contrôle et d'alerte dans la passation des marchés publics. 